\title{
Paths to the Adoption of Electric Vehicles: An Evolutionary Game Theoretical Approach
}

Sara Encarnação

(a) Interdisciplinary Centre of Social Sciences - CICS.NOVA - FCSH/UNL, Avenida de Berna, 26-C, 1069-061 Lisboa, Portugal

(b) INESC-ID and Instituto Superior Técnico, Universidade de Lisboa, IST-Taguspark, 2744-016 Porto Salvo, Portugal.

(c) ATP-group, P1649-003 Lisboa Codex, Portugal. sara.encarnacao@fcsh.unl.pt

Fernando P. Santos

(b) INESC-ID and Instituto Superior Técnico, Universidade de Lisboa, IST-Taguspark, 2744-016 Porto Salvo, Portugal.

(c) ATP-group, P1649-003 Lisboa Codex, Portugal. fernando.pedro@ tecnico.ulisboa.pt

Francisco C. Santos

(b) INESC-ID and Instituto Superior Técnico, Universidade de Lisboa, IST-Taguspark, 2744-016 Porto Salvo, Portugal.

(c) ATP-group, P1649-003 Lisboa Codex, Portugal. franciscocsantos@ist.utl.pt

Vered Blass

(d) Coller School of Management, Tel Aviv University, Tel Aviv 69978, Israel. vblass@ post.tau.ac.il

Jorge M. Pacheco

(e) Centro de Biologia Molecular e Ambiental and Departamento de Matemática e Aplicações, Universidade do Minho, 4710 - 057 Braga, Portugal.

(c) ATP-group, P1649-003 Lisboa Codex, Portugal.jmpacheco@math.uminho.pt

Juval Portugali

(f) ESLab Environmental Simulation Laboratory, Tel Aviv University, Tel Aviv 69978, Israel. juval@ post.tau.ac.il 


\begin{abstract}
Electric vehicles (EVs) are a viable alternative to internal combustion engine (ICE) vehicles, with the potential to alleviate the negative externalities stemming from the present ICE-based transportation sector. Notwithstanding, the current prevalence of ICE creates a lock-in state that averts the adoption of alternative and environmental friendly technologies, bringing forth a social dilemma. Here we investigate the feasibility of escaping the present lock-in state by studying possible incentive mechanisms involving, simultaneously, governments (public), companies (private) and consumers (civil). Resorting to Evolutionary Game Theory (EGT), we develop a theoretical model grounded on the strategic interactions between players from the different sectors, whose co-evolving choices influence (and are influenced by) different policies and social incentives. Our findings suggest that i) Public regulation is necessary but not sufficient for guaranteeing full EV adoption; ii) public-civil synergies are essential; iii) demand for EVs preceding supply is most efficient, providing companies with the needed incentives to counterweigh infrastructure investments; and iv) full adoption of EVs requires coordination between the three sectors to emerge, particularly when changes are initiated by the public sector.
\end{abstract}

Keywords: electric vehicles | evolutionary dynamics | diffusion | policy | game theory | 


\section{Introduction}

The contemporary means of transportation are mostly based on fossil fuels, thus constituting a chief source of air pollution and harmful environmental emissions (Colvile et al. 2001; Egbue and Long 2012; Grazi and van den Bergh 2008; Hoen et al. 2014). Changing to less polluting energy based transport technologies acquired recently a renewed focus, of which the transition from internal combustion engines (ICE) to electric vehicles (EVs) provides a paradigmatic example.

EVs are nowadays perceived as a solution to reduce environmental problems emerging from the road transport sector (Thiel et al. 2010). As in the past, the snag is, however, that status quo reflects the widespread dominance of ICE vehicles, which poses a coordination dilemma to alternative technologies (such as EVs) that remains unsolved to date.

Originating in the late XIX century, the automobile industry witnessed then a competition among several engine alternatives. From steam-powered vehicles to electric and gasoline, all were, at some point, a potential dominant technology (Anderson and Anderson 2010). In time, the gasoline vehicle prevailed and the industry grew into today's global scenario.

The ensuing success of the ICE car surpassed the technological domain and has since then reached deep into almost all domains of society. At the forefront of this societal conquest lies the continual technological development of the transportation sector (Barret 1996; Bruegmann 2006). In time, this self-reinforcing process has led the world, through positive feedbacks and path-interdependences (Cowan and Hultén 1996), to a lock-in state where the ICE engine constitutes the prevailing technology and any beneficial alternative has proved difficult to massively diffuse into the market. This state originated a social trap where seeking short-term benefits (e.g. accessibility and avoiding costs of changing the paradigm) generate negative collective externalities (e.g. air pollution) that seriously affect long-term wellbeing. This scenario, together with problems inherent to the EVs, leads to the following 
6 obstacles, identified as the main impediments to full adoption of EVs (Egbue and Long 2012; Giffi et al. 2011; USEPA 1996): 1) battery range and degradation, 2) higher purchasing costs, 3) limited charging infrastructure and fairly long recharging time, 4) perceived risks, 5) running costs and 6) the evolution of technology. Battery range and planning of charging infrastructures are complex problems still the focus of on-going research (Chen et al. 2016; Lee and Han 2017; Montoya et al. 2017; A. Zhang et al. 2017). Besides these barriers, adherence to EVs faces yet deeper issues related with individual and social perceptions regarding environmental prospects (Lorenzoni et al. 2007). These comprise lack of knowledge, uncertainty and skepticism (in technology and scientific outputs), delayed effects in time, reluctance to change lifestyles, personal control issues, lack of political action, freerider effects, social norms and expectations (Lorenzoni et al. 2007). All together, these perceptions create a "behavioral gap" in environmental consumerism that translates into a mismatch between pro-environmental attitudes of consumers and their willingness to engage in pro-environmental behavior, including buying products and services that minimize the impact on the environment (Gifford et al. 2011; Gupta and Ogden 2006; Moons and De Pelsmacker 2012; Schuitema et al. 2013; Steg 2005; Wu et al. 2010; J. Zhang et al. 2013). These technological and social barriers lead, in fact, to a tragedy of the commons type scenario, in which a public good (environmental quality), built upon social cooperation (here through EVs adoption), is endangered by the temptation to free ride (individual gain seeking) (Hardin 1968; Levin 2000). The complexity inherent to this type of social dilemma usually requires multi-level and multi-sectorial responses towards coordination into avoiding the non-cooperative state since, at this time, it is not clear whether surpassing technological barriers is sufficient to adopt new products, thus attaining new equilibrium states. This is particularly relevant given the way that individuals discount the future, being unable to incur immediate costs to trigger future benefits (Levin 2012; Van Lange et al. 2013). Several 
forecasting models try to understand and predict the impact of EVs adoption on market shares (Glerum et al. 2014; Jensen et al. 2017; Plötz et al. 2014), where it becomes clear the role of the above barriers on consumers' choices. Here we adopt a different approach, including not only the behavior of the demand side but also how this behavior might impact, and be impacted by, the supply side and the public sector (the ultimate player in promoting policies to deal with collective social problems).

The interrelation between societal sectors that exhibit multiple (and potentially diverging) interests produces an incentive landscape that is often challenging to analyze in a systematic way, despite its pervasiveness (Encarnação et al. 2016; Santos et al. 2016). Furthermore, individual preferences across different sectors are dynamically intertwined, as they are influenced by the actions performed, in a given moment, by the representatives of all other sectors. This multi-sectorial and frequency dependent nature of incentives, typically disregarded in conventional forecasting models, falls naturally in the framework of ecological modeling, where situations involving multiple species such as predator-prey dynamics or symbiotic interactions are well-known. In this way, the methods used in theoretical ecology can be employed to better understand the co-evolution of behaviors in socio-economic systems (May et al. 2008; Skyrms 1996) such as the present one underlying EVs adoption. Evolutionary Game Theory (EGT) (Hofbauer and Sigmund 1998; Smith 1982; Weibull 1995) constitutes, in this context, the mathematical framework of choice that builds on the idea of strategic interaction present in classical Game Theory (GT), yet relaxing the rationality assumptions often made there. By resorting to EGT, we explore here the similarities between the principles that govern strategy adoption and peer-influence in social systems and those governing trait evolution through natural selection: successful behaviors (similar to genes) spread faster within populations. This is apparent in the case of innovation diffusion (Rogers 2003) in which embracing new products is both a combination of 
spontaneous exploration (mutations or adoption by innovators) and success imitation (social learning or adoption by imitators). In the specific case of EVs, it is likely that the adoption of this technology follows the same principles of multi-sectorial frequency dependence, exploration and peer-influence: governmental subsidies, shared infrastructure costs, technological investment or environmental activism (to name a few) are mechanisms whose implementation depends on the engagement of individuals from particular sectors, and which, if effective, may drive the will of other sectors. These feedback loops, in turn, may steer early adoption and social contagion processes that - similarly to other technological innovations - may determine the disengagement of ICE vehicles and the overall adoption of EVs (Mercure et al. 2014).

Overall, our contribution to transportation research literature is in applying an analytical framework, based on a population dynamics EGT model, to explore the possibility of escaping the aforementioned lock-in state, where strong interdependencies among, public, private and civil players are present.

\section{Modelling Framework}

Following (Encarnação et al. 2016), we consider three populations: the public, private and civil sectors. The public sector represents government entities, the private sector businesses that produce and/or sell vehicles and the civil sector represents consumers.

Players in each population (public/private/civil) can adopt one of two strategies: To be in favor of electric vehicles, labeled as cooperators (C), or maintain the present status quo of ICE dominance, labeled as defectors (D). The individual payoff earned in one interaction (or encounter) depends on the strategy of each participant in each sector. An encounter occurs between three players, each belonging to a different population, and the resultant payoffs are arranged in the matrix/table detailed below. The dynamical process of behavior adoption 
assumes that the frequency-dependent fitness (average payoff) of each strategy will determine the future adoption of that same strategy, complying with an adaptive scheme akin to social learning (Rendell et al. 2010).

\subsection{Payoff matrix and parameters}

The individual payoffs and associated parameters are given in Tables 1 and 2, reflecting the interdependences within and between populations.

\begin{tabular}{lc}
\hline Game parameters & Symbol \\
& \\
\hline Public incentives in the form of subsidies & $S$ \\
Share of $S$ received by the private sector & $\delta$ \\
Cost of recharging infrastructures & $c_{i}$ \\
Share of $c_{i}$ imputed to the public sector & $\rho$ \\
Coordination benefit of selling/buying a vehicle & $b$ \\
\hline $\begin{array}{l}\text { Increments to EV technology } \\
\text { Punishment imposed on the private sector by citizen }\end{array}$ & $P_{b}$ \\
Activism and product boycotting & \\
Punishment imposed on the public sector through & $P$ \\
Citizen activism & \\
Public-civil synergistic effects & $\Delta 1$ \\
Private-civil synergistic effects & $\Delta 2$ \\
Green taxes over the benefits of private defectors & $\gamma$ \\
\hline
\end{tabular}

Table 1. Game parameters.

\begin{tabular}{cccccc}
\multicolumn{2}{c}{$\begin{array}{c}\text { Strategies } \\
\text { Public }\end{array}$} & Private & Civil & Public & $\begin{array}{c}\text { Payoffs accruing to each player } \\
\text { Private }\end{array}$ \\
C & C & C & $-S-\rho c_{i}$ & $S \delta+b-(1-\rho) c_{i}$ & $(1-\delta) S+b+i$ \\
C & C & D & $-S-\rho c_{i}$ & $S-(1-\rho) c_{i}$ & 0 \\
C & D & C & $\Delta 1-c_{i}$ & $-P_{b}$ & $(z-1) P_{b}+\Delta 1$ \\
C & D & D & $b \gamma-c_{i}$ & $b(1-\gamma)$ & $b$ \\
D & C & C & $-P$ & $\Delta 2+b-c_{i}$ & $(z-1) P+\Delta 2+b+i$ \\
D & C & D & 0 & $-c_{i}$ & 0 \\
D & D & C & $-P$ & $-P_{b}$ & $(z-1)\left(P+P_{b}\right)$ \\
D & D & D & 0 & $b$ & $b$
\end{tabular}

Table 2. Payoff table, where $\mathrm{C}$ indicates a cooperator and $\mathrm{D}$ a defector.

The central problem under study is to understand how to solve the social dilemma encoded in Table 2, that is, how to promote all sectors to switch to strategy $\mathrm{C}$, corresponding to a 
(future) better environmental quality. As such, the payoff table above must include those terms that accrue to processes that occur in a time-scale compatible with the decision-making process. Thus, the set of incentives that we consider here and that determine EV or ICE adoption - listed in Table 1 and included in Table 2 - interrelate in the following way:

- When supporting the adoption of EVs, public cooperators provide incentives in the form of subsidies $(S)$ shared between private $(\delta \in[0-1])$ and civil $(1-\delta)$ cooperators, following empirical examples where different policy designs exist (Dijk et al. 2013; Lévay et al. 2017). Subsidies will incur in a cost to the public sector and a benefit to both private and civil sectors. Subsidies can include tax exemptions or direct subsidies as a means to improve the cost-competitiveness of EVs, by lowering their total cost of ownership (Lévay et al. 2017). On the supply side, subsidies can take the form of, for example, R\&D grants to support technological innovation (EERE 2018; IEA 2018) .

- EVs require the development of specific recharging infrastructures. It is not clear, at present, who should initially pay for this development. Therefore, infrastructure costs $\left(c_{i}\right)$ can be imputed to both public $(\rho \in[0,1])$ and private $(1-\rho)$ cooperators.

- For simplicity, we assume that the private sector will only sell vehicles when encountering a consumer (civil sector) with the same strategy (whether C or D). As such, each will receive a benefit $(b)$ for finding a corresponding match in the market and selling/buying a car. As such, this benefit will not distinguish between EVs and ICEs cars.

- The model incorporates a positive dependence on technology that mimics increments to EV technology $(i)$. Thus, it is assumed that lower technological uncertainty translates into increased benefits for civil cooperators when buying an electric 
vehicle, and consequently, improves the feasibility of escaping the lock-in state (Berckmans et al. 2017).

- When private and civil players meet but each adopts a different strategy (C or D), no transaction occurs since the type of vehicle for sale does not correspond to the preferred type of vehicle to buy, and vice-versa. Thus, neither will receive the corresponding benefits. If the civil agent is a defector, and meets a private cooperator, his/hers derived payoffs will be null since no transaction occurs and civil defectors, as opposed to civil cooperators, do not incur in any activism costs (e.g. punishment or boycott) since they do not have to actively seek for the continuity of the status quo (i.e. ICE dominance). However, for the private agent these payoffs will include the cost of infrastructure development $\left(c_{i}\right)$. This burden can be alleviated if, as noted above, a public cooperator is present and shares part of these costs $(\rho)$ and, simultaneously, subsidizes the private cooperator.

- When the strategies adopted are reversed, i.e. a civil cooperator meets a private defector, then he/she can resort to boycott $\left(P_{b}\right)$, as a form of social punishment to private defectors (Franks et al. 2014; Harris and Jung-a 2017) as a way to induce change of private manufactures behavior (Kennedy 2017). Boycott is costly to civil cooperators but will decrease as the number of civil individuals with the same strategy increases (i.e., it will be frequency dependent). As before, the presence of a public cooperator can counterbalance these costs since both public and civil cooperators can share synergistic effects $(\Delta 1)$. Synergies can be understood as an effort to tackle negative externalities (e.g. noise and air pollution) reflecting the alignment between the public and the civil sectors. Examples of these synergies are low emission zones and congestions charges, such as, for example, those implemented in London, Stockholm, Lisbon (CML 2018a; Eliasson 2014; Styrelsen 2018; TFL 2018), velocity 
limits in specific city areas (CML 2018b), emission reduction targets for ICE vehicles (Commission 2018), etc. The implementation of the above policy measures target the reduction of the negative externalities associated with ICE vehicles thus inducing positive synergies for both government and individuals.

- On the regulation side, when only ICE cars are produced/sold and bought (private and civil defectors), public cooperators will try to promote the adoption of EVs through: 1) the introduction of "green" taxes $(\gamma)$ over the benefit of private defectors and 2) by developing the necessary re-charging infrastructure and paying a fraction $(\rho)$ of the corresponding costs $\left(c_{i}\right)$.

- On the other hand, if the public sector adopts the defector strategy, these costs will be entirely paid by private cooperators, when encountering a civil cooperator. Private cooperators can lessen these costs by 1 ) collecting the benefit (b) of selling EVs and by 2 ) sharing synergistic effects with civil cooperators $(\Delta 2)$. These synergies can include car-sharing schemes, such as Drive Now (DriveNow 2018), marketing for target sub-divisions of the consumer market and its social network (Axsen and Kurani 2012; Roger et al. 2016), free apps of journey assistance to help reduce range anxiety (Roger et al. 2016). Simultaneously, these synergies will contribute to the reduction of the costs of those civil cooperators engaging in social punishment to the public sector $(P)$ - which, as with boycotting, will depend on the frequency of civil cooperators. Social punishment can take the form of direct losses in public support to government, as for example elections or public protests that can influence public policy (Carter and Jacobs 2014; Hobolt and Klemmemsen 2005; Steinhardt and Wu 2016) or of indirect losses as the spread of negative perceptions on air quality and related health risks through media and social media contents (Wang et al. 2015). 


\subsection{Population dynamics}

We consider three different populations of finite sizes $Z_{\text {private }}=Z_{\text {public }}=Z_{\text {civil }}=Z$. The dynamics emerging from the 3-sector interactions are analyzed through an Evolutionary Game Theory (EGT) framework, where the adoption of strategies (C or D) within each sector follows a fitness dependent birth-death process implemented for 3 finite populations. This method mimics the social learning that occurs within human societies whenever successful behaviors of role models are widely adopted by other individuals (Rendell et al. 2010). It also allows one to model a process of social contagion, especially significant in new technologies without a mature market (Jensen et al. 2017). Conveniently, the frequency of each strategy (C or D) in the 3 populations defines a possible state of our system, in such a way that all the possible states can be represented by means of a cube (Figure 1 below). Each point (x,y,z) of that cube matches a state in which a fraction $\mathrm{x}(\mathrm{y}, \mathrm{z})$ of the individuals of the public (private, civil) population cooperate. In this way, the vertexes comprise the configurations where each population is either full cooperating (C) or full defecting (D). The fitness (or success) of an individual adopting a strategy $\mathrm{X}$ within a sector is given by the average return obtained from the 3-player game defined by the matrix of Table 2. Each game involves an individual randomly selected from each of the 3 populations, leading to an average payoff given by

$$
\begin{aligned}
& f_{X}^{\text {Public }}(x, y, z)=y z P_{X C C}^{\text {Public }}+\left(\begin{array}{ll}
1 & y
\end{array}\right) z P_{X D C}^{\text {Public }}+y(1 \quad z) P_{X C D}^{\text {Public }}+\left(\begin{array}{lll}
1 & y
\end{array}\right)(1 \quad z) P_{X D D}^{\text {Public }} \\
& f_{X}^{\text {Private }}(x, y, z)=x z P_{C X C}^{\text {Private }}+\left(\begin{array}{ll}
1 & x
\end{array}\right) z P_{D X C}^{\text {Private }}+x\left(\begin{array}{ll}
1 & z
\end{array}\right) P_{C X D}^{\text {Private }}+\left(\begin{array}{lll}
1 & x
\end{array}\right)\left(\begin{array}{ll}
1 & z
\end{array}\right) P_{D X D}^{\text {Private }} \quad \text { and } \\
& f_{X}^{\text {Civil }}(x, y, z)=x y P_{C C X}^{\text {Civil }}+\left(\begin{array}{lll}
1 & x
\end{array}\right) y P_{D C X}^{\text {Civil }}+x(1 \quad y) P_{C D X}^{\text {Civil }}+\left(\begin{array}{lll}
1 & x
\end{array}\right)(1 \quad y) P_{D D X}^{\text {Civil }} .
\end{aligned}
$$

where $f_{X}^{\mathrm{S}}(x, y, z)$ conveys the average payoff of an individual from sector $\mathrm{S}$ while adopting strategy $\mathrm{X}$, provided that the population is currently in the point $(\mathrm{x}, \mathrm{y}, \mathrm{z}) . P_{A B C}^{\mathrm{S}}$ translates the payoff obtained by an individual from state $S$ when playing in a group with the strategy 
profile where Public plays A, Private plays B and Civil plays C. These payoffs follow Table 2.

As in (Encarnação et al. 2016), we assume two mechanisms of strategy update: imitation and mutation. A mutation is said to occur whenever an individual adopts, spontaneously and without resorting to any imitation process, a new strategy. Conversely, imitation (or social learning) requires 1) the imitated strategy to be already present in the population and 2) a fitness value associated with that strategy. Here we adopt the so-called pairwise comparison rule (Traulsen et al. 2006), where the successful individuals tend to be imitated more often and their strategy spread within each sector. At each time step, a random individual $i$ may imitate another individual $j$ randomly selected from the same sector. This imitation occurs with a probability $p$ that increases with the fitness difference between the role model $j$ and the imitator $i$. We assume that $p=\left[1+e^{\left[f_{j} f_{i}\right]}\right]^{1}$, where $f_{j}$ and $f_{i}$ are the fitness values of individuals $i$ and $j$, respectively, such that an imitator will have a higher probability of imitating a model that reveals a comparatively higher fitness. The parameter $\beta$ represents the "selection pressure" or "intensity of selection", i.e., the significance of individual fitness in the decision to imitate. For $\beta<<1$, selection is weak, such that for $\beta=0$ the decision to imitate is randomly adopted. For large $\beta$, imitation becomes increasingly deterministic. As it is often the case in natural and human social dynamics (Bell 2010; Sigmund 2010; Traulsen et al. 2010), here we resort to strong selection $(\beta=1)$, yet allowing for errors in decision-making, potentially induced by confounding factors common to Human decision-making, and that often translates into a bounded rational behaviors of players (Gigerenzer and Selten 2002; Simon 1987).

Our analysis can be considerably simplified whenever we adopt the well-known small mutation approximation (Fudenberg and Imhof 2006) adapted for a multi-sector paradigm (Encarnação et al. 2016). In this way we reduce the state space under analysis, while still 
keeping vital information regarding the overall dynamics in the full-state space. Technically, this corresponds to a maximal reduction of the state space in a hierarchy of approximations (Vasconcelos et al. 2017). The intuition behind this approximation can be realized by noticing that, under a process of imitation dynamics, only a new mutation can either introduce a new strategy or bring back a strategy that has become extinct. If new strategies (i.e., not yet existing in the population) are rarely introduced - a situation occurring for low mutation probabilities, as detailed above - the population will naturally evolve towards a state where all individuals in each sector adopt the same strategy (so-called monomorphic). While a monomorphic state will, in this case, always be reached, we do not make any considerations regarding the time needed for that to occur. Thus, when a (rare) mutation occurs, the system leaves a monomorphic state, and one out of two scenarios can happen: 1) the mutant strategy is successively adopted in the corresponding sector, until a new monomorphic state is reached or 2) the mutation is not successful and the system returns to the previous monomorphic state. Assuming that mutations are sufficiently rare means that it is reasonable to assume that no other mutations - particularly in other sectors - occur until one of these scenarios is verified. In this low-mutation regime, the evolutionary process can thus be analyzed by calculating the evolutionary dynamics in each sector alone. To this end, we compute the fixation probabilities $\left(\rho_{a b}\right)$, that is, the probability that a single mutant with a strategy $b$ (either $\mathrm{C}$ or D) fully invades a population of Z-1 individuals using the opposite strategy $a$ (Nowak et al. 2004),

$$
\rho_{a b}=\left(1+\sum_{i-1}^{Z-1} \prod_{j=1}^{i} \frac{T_{j}^{-}}{T_{j}^{+}}\right)^{-1}
$$

where $T_{j}^{+}\left(T_{j}^{-}\right)$represents the probability that one more individual adopts strategy $b$ in a population of $j$ individuals already using that strategy $b$. The $T_{j}^{+}\left(T_{j}^{-}\right)$are calculated assuming 
the pairwise imitation process described above (Traulsen et al. 2006), and are given by $T_{j}^{ \pm}=\frac{j}{Z} \frac{Z \quad j}{Z}\left[1+e^{\square\left(f_{b} f_{a}\right)}\right]^{1}$

The aforementioned transition probabilities $\left(T_{j}^{+}\right.$and $\left.T_{j}^{-}\right)$, defined for any possible state $(\mathrm{x}, \mathrm{y}, \mathrm{z})$, also allow one to define a gradient of selection $\left(T_{j}^{+}-T_{j}^{-}\right)$indicating the most probable path in state space followed by the three populations, with respect to the adoption of strategies. By numerically integrating this gradient for a given initial condition, it is possible to follow the average trajectories that reflect the most probable evolutionary path (along the full state space) of our 3 populations (Figure 2 and 3 below). Trajectories in state space in which the composition of only one population changes are represented by the edges of the cube. Along each edge, selection favors the fixation of a $\mathrm{C}$ in a population of Ds over the fixation of a $\mathrm{D}$ in a population of Cs whenever $\rho_{C}>\rho_{D}$. In specific cases, this inequality also provides information regarding the eventual risk-dominance of each strategy (Nowak 2006).

We also compute the average fraction of time spent in each monomorphic state (the cube vertexes in Figures 2 and 3 below). To this end, we calculate the so-called stationary distribution (in the small mutation approximation), taking advantage of the Markov nature of the process, with transition probabilities given by the aforementioned fixation probabilities (and condensed in the transition matrix $\mathbf{T}$, with $\mathbf{T}_{a b}={ }_{a b} / 3\left(\begin{array}{ll}j & i\end{array}\right)$ and $\left.\mathbf{T}_{i i}=1 \quad \mathbf{T}_{a b}\right)$. Whenever, as is the case, the Markov chain is irreducible there is a unique stationary distribution ${ }^{*}$ calculated as the eigenvector (of matrix $\mathbf{T}$ ) associated with eigenvalue 1, i.e. ${ }^{*} \mathbf{T}={ }^{*}$ (Fudenberg and Imhof 2006; Vasconcelos et al. 2017).

\section{Results: Paths to the Adoption of Electric Vehicles}


Let us now analyze how the preferences of each sector co-evolve, which translates into different evolutionary trajectories that potentially lead to the overall adoption of EVs. The overall dynamics is illustrated in Figure 1, where it is clear that some transitions towards the adoption of EVs are likely to be fulfilled. This said, escaping the current lock-in state (in which everyone supports ICE, i.e., vertex DDD) requires the intervention of the public sector, as both private and civil sectors lack the required incentives to initialize the technological transition by themselves (see payoffs for transitions DDD $\rightarrow$ DCD and DDD $\rightarrow$ DDC in Table 2 above and edges 9 and 5 in Figure 1).

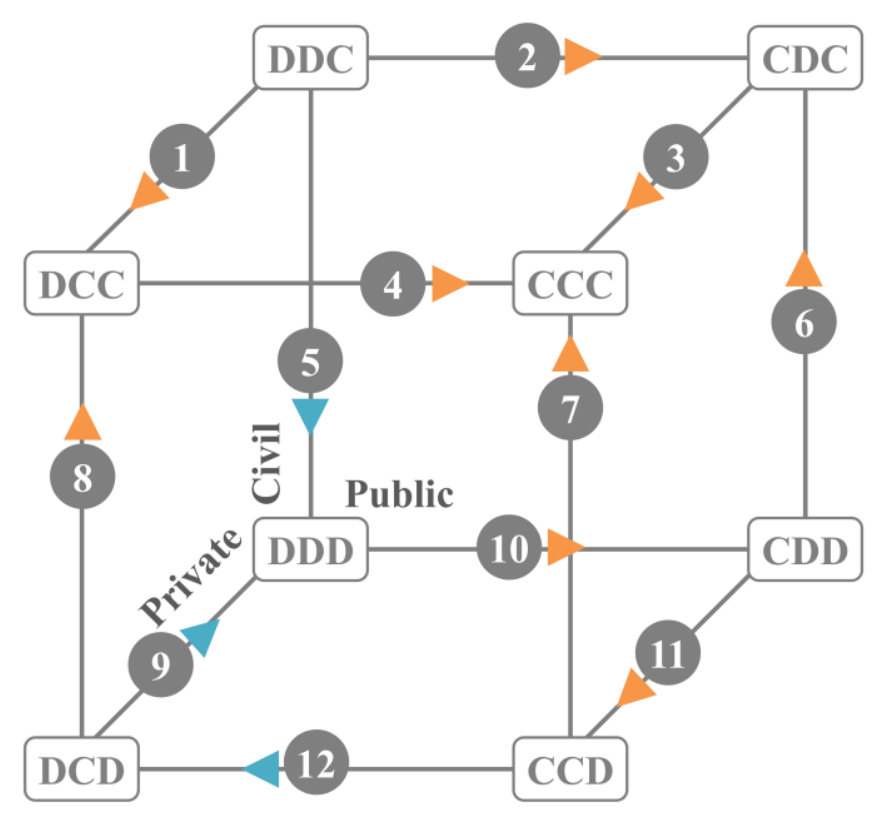

\section{Conditions for Cooperation}

$$
\begin{aligned}
& \text { 1) } b+\Delta 2-c_{i}>-P_{b} \\
& 2>\Delta 1-c_{i}>-P \\
& 3>b+S \delta-c_{i}(1-\rho)>-P_{b} \\
& 4>S+c_{i} \rho<P \\
& 6>\Delta 1-P_{b} / 2>b \\
& \text { 7) } b+i+S(1-\delta)>0 \\
& 8>b+i+\Delta 2>P / 2 \\
& \text { (10) } c_{i}<b \gamma \\
& \text { (1) } b(1-\gamma)<S-c_{i}(1-\rho)
\end{aligned}
$$

\section{Always defection}

Figure 1. Three player representation cube (Left). Each edge is accompanied by conditions (orange arrows, detailed in the Right panel) that, when verified, imply that the fixation of Cs in a population of Ds is higher than the fixation of Ds in a population of Cs. While here we focus on the analytical results that describe the transitions between states where only one sector changes its strategy, all other possible transitions (including through the interior of the cube) are later explored, in Figures 2 and 3, by employing numerical simulations. Note, however, that the above conditions which govern the edges of the cube will also influence the dynamics inside the cube.

In fact, by unilaterally promoting EVs adoption, private cooperators would need to deploy infrastructure development and pay for this costly enterprise. This extra investment may be discouraging (Yang and Holgaard 2012) and in some way expresses the propensity of 
manufacturers to absorb and control change (Wells and Nieuwenhuis 2012). On the other hand, by being the only sector to choose EVs, civil cooperators would have to bear the high costs of being misaligned with the private sector. Hence, regardless of the initial efforts from the private and civil sectors, unlocking state DDD requires intervention from the public sector. By, e.g., collecting "green" taxes, public cooperators can gain advantage over public defectors when these gains outweigh the cost of unilaterally providing the deployment of the necessary recharging infrastructure (edge 10 in Figure 1 and Figure 2).
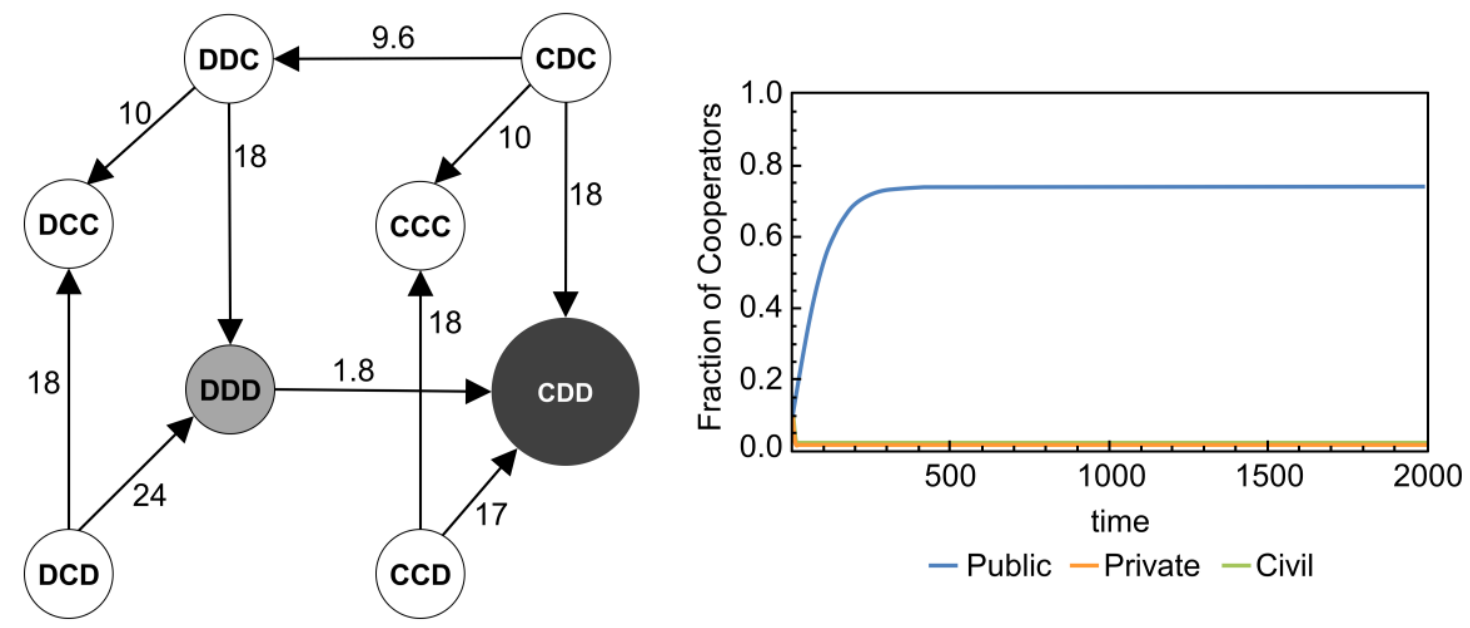

Figure 2. The evolutionary dynamics under study follows a Markov Process whose transition probabilities are pictured in a cube, in which vertices match the considered states (Left panel). The transition probabilities are normalized by $1 / Z$, the neutral drift probability. Only transitions with probability higher than $1 / \mathrm{Z}$ are represented. The diameter and color of the circles translates the fraction of time spent in those same states (provided by the stationary distribution - see section 2.2 above). The stochastic nature of dynamics suggests that there is not a unique time series corresponding to the time evolution of strategy adoption. Thereby, we represent the most probable time series, starting from a state in which $10 \%$ of each population adopts Cooperation (Right panel). Parameters' values: $S=0.0 ; \delta=0.5 ; c_{i}=0.24 ; \rho=0 ; b=0.5 ; i=0 ; P_{b}=0.0 ; P=0.0 ; \Delta 1=$ $0.0 ; \Delta 2=0.0 ; Z_{\text {civil }}=Z_{\text {public }}=Z_{\text {private }}=Z=50 ; \gamma=0.54$.

\subsection{Towards a stable state of EV adoption}

Once the public supports EVs, new incentive mechanisms can be implemented in order to steer the behavior of the other two sectors. The challenge is now to decide between acting on the supply side (private sector) or on the demand side (civil sector). On the supply side, subsidies given to private cooperators will have to compensate the benefit of selling ICE 
vehicles and also the costs of infrastructure development (see condition 11 in Figure 1). On the demand side (civil sector), the benefit of owing an ICE vehicle must be lower than the synergies with the public sector and these should be sufficient to compensate the cost of boycotting (condition 6 in Figure 1).

In Figure 3, we explore how EV adoption evolves in time, when emphasis is placed on these different public incentives. As can be seen in panel A, by first promoting the demand side through public-civil synergies $(\Delta 1=0.51)$, the world will reach state CCC. Here, and in the absence of subsidies $(S=0)$, punishment $(P=0)$ and infrastructure costs $(\rho=0)$, the world will drift between states DCC and CCC, since both strategies bring equal payoffs to public players. This shows that the public sector can withdraw its direct influence in the game when the private and civil sectors reach full adoption of EVs.

By including subsidies (panel B Figure 3), and equally distribute them between private and civil players $(\delta=0.5)$ it is possible to promote overall cooperation in these two populations. As subsidies represent a costly endeavor, once private and civil sectors coordinate into EV support, it is likely that the public sector retracts from such monetary burden (thus reaching state DCC as before). This withdrawal can be prevented if civil cooperators are willing to punish public defectors (panel C of Figure 3) or if high levels of public-civil synergies exist. In this case, the system will ultimately stabilize in state CCC. The trajectories in Figure 3 also show that combining different mechanisms (policy instruments) can contribute to speed up EV adoption, in accordance with empirical research on environmental policy performance (Daugbjerg and Sønderskov 2012) - see also (Encarnação et al. 2016). 

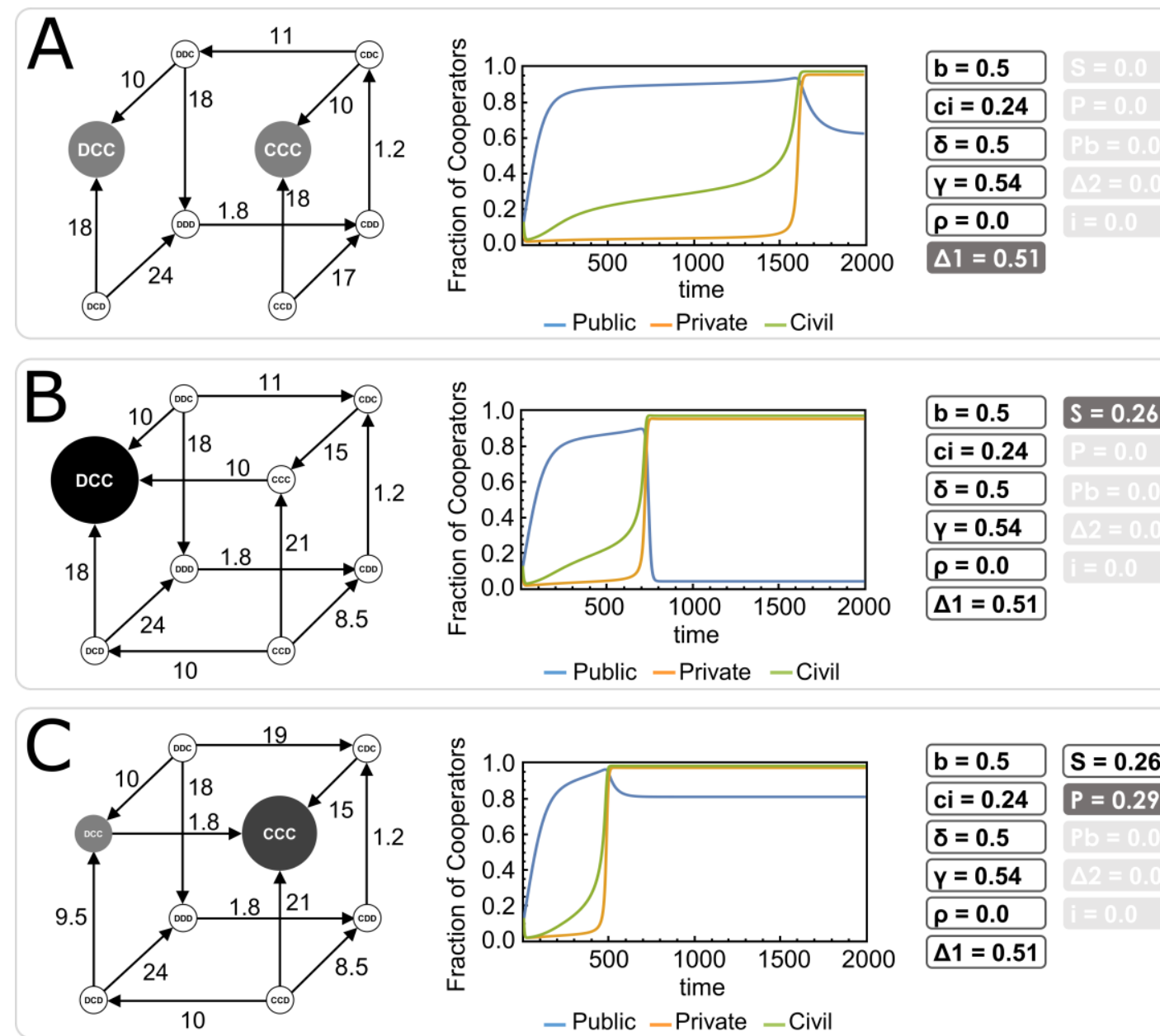

$S=0.26$

$P=0.29$

Figure 3. The evolutionary dynamics under study follows a Markov Process whose transition probabilities are pictured in a cube, in which vertices match the considered states (Left panels). The transition probabilities are normalized by $1 / Z$, the neutral drift probability. Only transitions with probability higher than $1 / \mathrm{Z}$ are represented; transitions with probability higher than $1 / \mathrm{Z}$ are said to be favored by natural selection. The diameter and color of the different states (circles) represent the fraction of time spent in those states. The stochastic nature of the dynamics suggests that there is no unique time series corresponding to the time evolution of strategy adoption. Thereby, we represent the most probable time series, starting from a state in which $10 \%$ of each population adopts a Cooperative (EV) strategy (Center panels). Panels A, B and C represent different combinations of parameters (see Right panels). As before, we assume $\mathrm{Z}=50$.

In what concerns those parameters not used in Figure 3 (that is, taken to be zero), namely civil boycott to private defectors $\left(P_{b}\right)$, private-civil synergies $(\Delta 2)$ and technological increments to EVs $(i)$, the conditions depicted in Figure 1 above show that:

- Being costly to civil cooperators, boycott $\left(P_{b}\right)$ can be avoided to the extent that publiccivil synergies $(\Delta 1)$ are in place. Examples of such synergies, in addition to the ones deemed previously, exist through regulatory standards, operational programs, 
information sharing and support to member activities in collaboration groups (Abbott 2012). The implicit cost associated with $P_{b}$ (e.g. in terms of time, learning and information gathering), renders it viable in scenarios where an engaged public sector is absent (defection, edges 1 and 8, cf. Figure 1). In these scenarios, private-civil synergies $(\Delta 2)$ can also push private and civil sectors into cooperation and can compensate the costs accruing to the private sector with infrastructure development $\left(c_{i}\right)$.

- Incremental developments in EVs technology $(i)$ will play a role in scenarios where the supply side is fostered first (edges 7 and 8, cf. Figure 1), an unlikely scenario in face of the results presented in this study. Nevertheless, the importance of this parameter becomes clear in shifting from a state where only private companies support EVs (vertex DCD, cf. Figure 1). When the benefits of buying EVs and private-civil synergies are not sufficient to compensate the costs of punishing, incremental development of EV technology can twist the balance and rapidly promote increasing numbers of civil cooperators towards the state DCC. This would prevent the private sector from changing to defection, thus leading to a demise of overall cooperation.

\section{Discussion and Conclusions}

Transition to EVs is a complex problem that extends beyond the competition between alternative technologies. At its core lies a social dilemma of collective action in which social benefits stemming from EV usage face the barrier of replacing a consolidated technology (ICE). By resorting to evolutionary game theory, we have developed a theoretical model to study the interdependences between three sectors of society, seeking to unveil the mechanisms that allow one to escape the dilemma and allow society to massively adopt EVs. 
Our findings suggest that the complexity inherent to EV adoption needs a proactive society with public support. Governmental regulation is determinant in providing the initial lead-off towards the transition to EV. This initial step can be self-enforced, as "green" taxes may provide the material incentive to counterbalance the advantage of the already established ICE vehicle. Once supporting EV, the public sector can activate mechanisms - such as subsidies - that may be used as financial backing of private and civil sectors. Once bootstrapped, the next question is whether one should opt between supporting the supply or the demand side. Our results show that demand should be the initial target and assured through public-civil synergies - a mechanism of paramount importance in our results.

Public-civil synergies can be understood as a way to increase environmental awareness and to raise the level of conformity between public and civil sectors. To guarantee this mutual support, some researches show that advertising campaigns on environmental benefits of green products can be relevant in changing behaviors (Nyborg et al. 2006). Public advertising may also contribute to bridging the gap between consumers intents/concerns and actions (Kalamas et al. 2014) which can increase their willingness-to-pay (Plötz et al. 2014) additional costs that are perceived as necessary to sustain long term collective gains (such as better environmental quality). Simultaneously, a strong and active civil sector (apt to influence public policy through synergies) can be more evident if individuals organize in social groups such as NGOs. While here we treat the civil sector as individual consumers, future work is needed in order to determine the viability of civil collective action and its influence on civil-public synergies.

In summary, our results show that the path to full adoption of EVs should start with public intervention, through subsidies, "green" taxes and synergies with the civil sector. The latter is determinant not only to foster a necessary demand side for EVs but also to compensate the costs of subsidizing both private and civil sectors. A more general interpretation of these 
results may hint on the relevance of these synergies in increasing the environmental awareness of the civil sector. A pro-environment and active population can not only express dissatisfaction through engagement (when needed) in consumer activism but can also contribute to shift valuations over the different products, putting a higher value on environmental friendly behavior than on the short-term benefit of owning an ICE vehicle. The increase demand for EVs could then pull the supply side towards full adoption of electric vehicles. We also highlight that, after the proper coordination of civil and private sectors into EV promotion, public intervention can be edged off without reducing the EV adoption levels. Finally, the present model highlights the importance of taking into consideration an increasingly organized civil society, a new third sector capable of disrupting the previously dominant two-player dilemma involving the public and private sectors.

\section{Competing financial interests}

The authors declare no competing financial interests.

\section{Acknowledgements}

This research is conducted in the frame of the project: "Diffusion of Mass E-Mobility and Integrating Renewable Energy in Smart Grids and Cities: Intelligent Agents for Efficient Energy Consumption”, funded by the Israeli Ministry of Energy and Water agreement 212-11-012 and with the financial support of Fundação para a Ciência e Tecnologia - FCT/MCTES through National funds, for project grants SFRH/BD/94736/2013 (FPS), SFRH/BPD/1169337/2016 (SE), PTDC/EEI-SII/5081/2014 (SE,FPS,FCS,JMP), PTDC/MAT/STA/3358/2014 (SE,FPS,FCS,JMP) and by multi-annual funding of CBMA, INESC-ID and CICS.NOVA (under the projects UID/BIA/04050/2013, UID/CEC/50021/2013 and UID/SOC/04647/2013) also provided by FCT/MCTES. 


\section{References}

Abbott, Kenneth W. (2012), 'Engaging the public and the private in global sustainability governance', International Affairs, 88 (3), 543-64.

Anderson, Curtis D. and Anderson, Judy (2010), Electric and Hybrid Cars: A History (2 edition edn.; Jefferson, N.C: McFarland) 267.

Axsen, Jonn and Kurani, Kenneth S (2012), 'Interpersonal Influence within Car Buyers' Social Networks: Applying Five Perspectives to Plug-in Hybrid Vehicle Drivers', Environment and Planning A: Economy and Space, 44 (5), 1047-65.

Barret, George (1996), 'The Transport Dimension', in Elizabeth Burton, Mike Jenks, and Katie Williams (eds.), The Compact City. A Sustainable Urban Form? (London ; New York: Routledge).

Bell, Graham (2010), 'Fluctuating selection: the perpetual renewal of adaptation in variable environments', Philosophical Transactions of the Royal Society B: Biological Sciences, 365 (1537), 87-97.

Berckmans, Gert, et al. (2017), 'Cost Projection of State of the Art Lithium-Ion Batteries for Electric Vehicles Up to 2030', Energies, 10 (9), 1314.

Bruegmann, Robert (2006), Sprawl: A Compact History (Chicago, Ill.: University Of Chicago Press) 306.

Carter, Neil and Jacobs, Michael (2014), 'EXPLAINING RADICAL POLICY CHANGE: THE CASE OF CLIMATE CHANGE AND ENERGY POLICY UNDER THE BRITISH LABOUR GOVERNMENT 2006-10', Public Administration, 92 (1), 125-41.

Chen, Zhibin, He, Fang, and Yin, Yafeng (2016), 'Optimal deployment of charging lanes for electric vehicles in transportation networks', Transportation Research Part B: Methodological, 91, 344-65.

CML 'Low Emission Zones', <http://www.cm-lisboa.pt/en/living-in/mobility/low-emissionzones $>$, accessed 23/01/2018.

--- '30km/h Zones', <http://www.cm-lisboa.pt/en/living-in/mobility/30-kmh-zones $>$, accessed 23/01/2018.

Colvile, R. N., et al. (2001), 'The transport sector as a source of air pollution', Atmospheric Environment, 35 (9), 1537-65.

Commission, European 'Reducing $\mathrm{CO} 2$ emissions from passenger cars', $<$ https://ec.europa.eu/clima/policies/transport/vehicles/cars_en $>$, accessed 23/01/2018.

Cowan, Robin and Hultén, Staffan (1996), 'Escaping lock-in: The case of the electric vehicle', Technological Forecasting and Social Change, 53 (1), 61-79.

Daugbjerg, Carsten and Sønderskov, Kim Mannemar (2012), 'Environmental Policy Performance Revisited: Designing Effective Policies for Green Markets', Political Studies, 60 (2), 399-418.

Dijk, Marc, Orsato, Renato J., and Kemp, René (2013), 'The emergence of an electric mobility trajectory', Energy Policy, 52, 135-45.

DriveNow 'DriveNow Car Sharing \& Car Club', <https://www.drive-now.com/en/>, accessed 23/01/2018.

EERE 'Small Business Innovation Research and Small Business Technology Transfer Programs', <https://energy.gov/eere/technology-to-market/small-businessinnovation-research-and-small-business-technology-transfer>, accessed 23/01/2018.

Egbue, Ona and Long, Suzanna (2012), 'Barriers to widespread adoption of electric vehicles: An analysis of consumer attitudes and perceptions', Energy Policy, 48, 717-29. 
Eliasson, Jonas (2014), 'The role of attitude structures, direct experience and reframing for the success of congestion pricing', Transportation Research Part A: Policy and Practice, 67, 81-95.

Encarnação, Sara, et al. (2016), 'Paradigm shifts and the interplay between state, business and civil sectors', Royal Society Open Science, 3 (12).

Franks, Daniel M., et al. (2014), 'Conflict translates environmental and social risk into business costs', Proceedings of the National Academy of Sciences, 111 (21), 757681.

Fudenberg, Drew and Imhof, Lorens A. (2006), 'Imitation processes with small mutations', Journal of Economic Theory, 131 (1), 251-62.

Giffi, Craig, et al. (2011), 'Unplugged: Electric vehicle realities versus consumer expectations', (Deloitte Touche Tohmatsu Limited Global Manufacturing Industry group), 32.

Gifford, Robert, Kormos, Christine, and McIntyre, Amanda (2011), 'Behavioral dimensions of climate change: drivers, responses, barriers, and interventions', Wiley Interdisciplinary Reviews: Climate Change, 2 (6), 801-27.

Gigerenzer, Gerd and Selten, Reinhard (2002), Bounded rationality: The adaptive toolbox (MIT press).

Glerum, Aurélie, et al. (2014), 'Forecasting the Demand for Electric Vehicles: Accounting for Attitudes and Perceptions', Transportation Science, 48 (4), 483-99.

Grazi, Fabio and van den Bergh, Jeroen C. J. M. (2008), 'Spatial organization, transport, and climate change: Comparing instruments of spatial planning and policy', Ecological Economics, 67 (4), 630-39.

Gupta, Shruti and Ogden, Denise T. (2006), 'The Attitude - Behavior Gap in Environmental Consumerism', APUBEF 29th Annual Meeting (3), 199-206.

Hardin, Garrett (1968), 'The Tragedy of the Commons', Science, 162 (3859), 1243-48.

Harris, Bryan and Jung-a, Song (2017), 'Hyundai Motor pushes electric car plans as sales slump', Financial Times, 1 June 2017.

Hobolt, Sara Binzer and Klemmemsen, Robert (2005), 'Responsive Government? Public Opinion and Government Policy Preferences in Britain and Denmark', Political Studies, 53 (2), 379-402.

Hoen, Kristel M. R., et al. (2014), 'Switching Transport Modes to Meet Voluntary Carbon Emission Targets', Transportation Science, 48 (4), 592-608.

Hofbauer, Josef and Sigmund, Karl (1998), Evolutionary games and population dynamics (Cambridge University Press).

IEA 'Policies and Legislation', <http://www.ieahev.org/by-country/>, accessed 23/01/2018.

Jensen, Anders F., et al. (2017), 'Predicting the Potential Market for Electric Vehicles', Transportation Science, 51 (2), 427-40.

Kalamas, Maria, Cleveland, Mark, and Laroche, Michel (2014), 'Pro-environmental behaviors for thee but not for me: Green giants, green Gods, and external environmental locus of control', Journal of Business Research, 67 (2), 12-22.

Kennedy, Christopher (2017), 'Comment: Boycott products from states with dirty energy', Nature, 551, 294-95.

Lee, Chungmok and Han, Jinil (2017), 'Benders-and-Price approach for electric vehicle charging station location problem under probabilistic travel range', Transportation Research Part B: Methodological, 106, 130-52.

Lévay, Petra Zsuzsa, Drossinos, Yannis, and Thiel, Christian (2017), 'The effect of fiscal incentives on market penetration of electric vehicles: A pairwise comparison of total cost of ownership', Energy Policy, 105, 524-33. 
Levin, Simon (2000), Fragile Dominion: Complexity and the Commons (First Paperback Printing, May 2000 edition edn.; Cambridge, Mass: Basic Books) 272.

--- (2012), 'The Trouble of Discounting Tomorrow', Solutions, 3 (4), 5.

Lorenzoni, Irene, Nicholson-Cole, Sophie, and Whitmarsh, Lorraine (2007), 'Barriers perceived to engaging with climate change among the UK public and their policy implications', Global Environmental Change, 17 (3-4), 445-59.

May, Robert M., Levin, Simon A., and Sugihara, George (2008), 'Complex systems: Ecology for bankers', Nature, 451 (7181), 893-95.

Mercure, J. F., et al. (2014), 'The dynamics of technology diffusion and the impacts of climate policy instruments in the decarbonisation of the global electricity sector', Energy Policy, 73, 686-700.

Montoya, Alejandro, et al. (2017), 'The electric vehicle routing problem with nonlinear charging function', Transportation Research Part B: Methodological, 103, 87-110.

Moons, Ingrid and De Pelsmacker, Patrick (2012), 'Emotions as determinants of electric car usage intention', Journal of Marketing Management, 28 (3-4), 195-237.

Nowak, Martin A. (2006), Evolutionary Dynamics: Exploring the Equations of Life (Canada: Belknap Press) 384.

Nowak, Martin A., et al. (2004), 'Emergence of cooperation and evolutionary stability in finite populations', Nature, 428 (6983), 646-50.

Nyborg, Karine, Howarth, Richard B., and Brekke, Kjell Arne (2006), 'Green consumers and public policy: On socially contingent moral motivation', Resource and Energy Economics, 28 (4), 351-66.

Plötz, Patrick, Gnann, Till, and Wietschel, Martin (2014), 'Modelling market diffusion of electric vehicles with real world driving data — Part I: Model structure and validation', Ecological Economics, 107, 411-21.

Rendell, L., et al. (2010), 'Why Copy Others? Insights from the Social Learning Strategies Tournament', Science, 328 (5975), 208-13.

Roger, Bennett, Rita, Kottasz, and Stephen, Shaw (2016), 'Factors potentially affecting the successful promotion of electric vehicles', Journal of Social Marketing, 6 (1), 62-82.

Rogers, Everett M. (2003), Diffusion of Innovations, 5th Edition (5th edition edn.; New York: Free Press) 576.

Santos, Fernando, et al. (2016), 'An Evolutionary Game Theoretic Approach to MultiSector Coordination and Self-Organization', Entropy, 18 (4), 152.

Schuitema, Geertje, et al. (2013), 'The role of instrumental, hedonic and symbolic attributes in the intention to adopt electric vehicles', Transportation Research Part A: Policy and Practice, 48, 39-49.

Sigmund, Karl (2010), The Calculus of Selfishness (1St Edition edition edn., Princeton Series in Theoretical and Computational Biology; Princeton: Princeton University Press) 184.

Simon, H (1987), 'Models of man, continuity in administrative science', (Ancestral Books in the Management of Organizations).

Skyrms, Brian (1996), Evolution of Social Contract (1st edn.: Cambridge University Press) 162.

Smith, John Maynard (1982), Evolution and the Theory of Games (1 edition edn.; Cambridge ; New York: Cambridge University Press) 234.

Steg, Linda (2005), 'Car use: lust and must. Instrumental, symbolic and affective motives for car use', Transportation Research Part A: Policy and Practice, 39 (2-3), 147-62.

Steinhardt, H. Christoph and Wu, Fengshi (2016), 'In the Name of the Public: Environmental Protest and the Changing Landscape of Popular Contention in China', The China Journal, 75, 61-82. 
Styrelsen, Transport 'Congestion taxes in Stockholm and Gothenburg', $<$ https://transportstyrelsen.se/en/road/Congestion-taxes-in-Stockholm-andGoteborg/>, accessed 23/01/2018.

TFL 'Congestion Charge', <https://tfl.gov.uk/modes/driving/congestion-charge>, accessed 23/01/2018.

Thiel, Christian, Perujo, Adolfo, and Mercier, Arnaud (2010), 'Cost and CO2 aspects of future vehicle options in Europe under new energy policy scenarios', Energy Policy, 38 (11), 7142-51.

Traulsen, Arne, Nowak, Martin A., and Pacheco, Jorge M. (2006), 'Stochastic dynamics of invasion and fixation', Physical Review E, 74 (1), 011909.

Traulsen, Arne, et al. (2010), 'Human strategy updating in evolutionary games', Proc Natl Acad Sci USA, 107 (7), 2962-66.

USEPA (1996), 'Indicators of the Environmental Impacts of Transportation. Highway, Rail, Aviation, and Maritime Transport', Policy, Planning and Evaluation (US: US Environmental Protection Agency), 268.

Van Lange, Paul A. M., et al. (2013), 'The psychology of social dilemmas: A review', Organizational Behavior and Human Decision Processes, 120 (2), 125-41.

Vasconcelos, Vítor V., et al. (2017), 'Stochastic Dynamics through Hierarchically Embedded Markov Chains', Physical Review Letters, 118 (5), 058301.

Wang, Shiliang, Paul, Michael J., and Dredze, Mark (2015), 'Social Media as a Sensor of Air Quality and Public Response in China', Journal of Medical Internet Research, 17 (3), e22.

Weibull, Jörgen W. (1995), Evolutionary Game Theory (US: The MIT Press) 265.

Wells, Peter and Nieuwenhuis, Paul (2012), 'Transition failure: Understanding continuity in the automotive industry', Technological Forecasting and Social Change, 79 (9), 1681-92.

Wu, Hsin-Ying, Trappey, Charles V., and Feinberg, Richard A. (2010), 'The Diffusion of Innovation and perceived risk for the adoption of alternative energy vehicles', International Journal of Innovation and Learning, 8 (3), 296-315.

Yang, Yan and Holgaard, Jette Egelund (2012), 'The important role of civil society groups in eco-innovation: a triple helix perspective', Journal of Knowledge-based Innovation in China, 4 (2), 132-48.

Zhang, Anpeng, Kang, Jee Eun, and Kwon, Changhyun (2017), 'Incorporating demand dynamics in multi-period capacitated fast-charging location planning for electric vehicles', Transportation Research Part B: Methodological, 103, 5-29.

Zhang, Jie, Kørnøv, Lone, and Christensen, Per (2013), 'Critical factors for EIA implementation: Literature review and research options', Journal of Environmental Management, 114, 148-57. 